\title{
Beyond the Nanomaterials Approach: Influence of culture conditions on the stability and antimicrobial activity of silver nanoparticles
}

\section{Supplementary material}

\author{
Roberto Vazquez-Muñoz ${ }^{1,2}$, Nina Bogdanchikova² and Alejandro Huerta-Saquero, ${ }^{2}{ }^{*}$ \\ ${ }^{1}$ Department of Biology and The South Texas Center for Emerging Infectious Diseases, The University of Texas at \\ San Antonio. One UTSA Circle, San Antonio, TX 78249, USA \\ 2 Centro de Nanociencias y Nanotecnología, Universidad Nacional Autónoma de México, Km 107 Carretera \\ Tijuana-Ensenada. CP 22860, Ensenada, Baja California, México.
}

email: saquero@cnyn.unam.mx 
Figure S1. Culture media alter the UV-Vis spectra of the AgNPs.
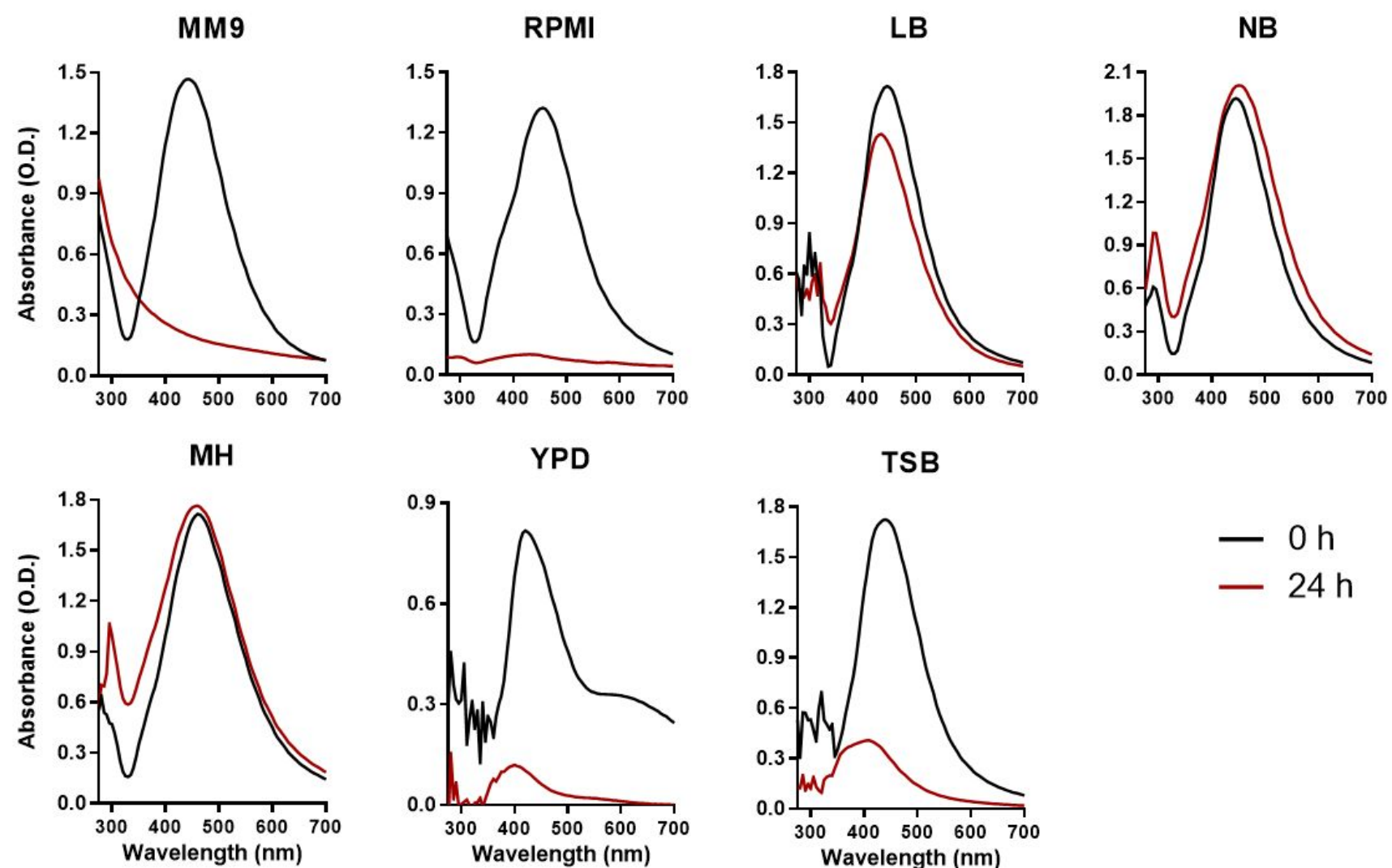

TSB
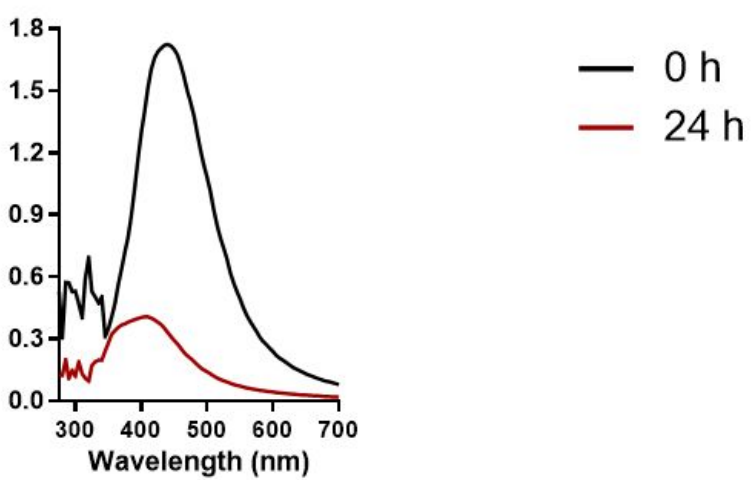

Figure S1. Culture media alter the UV-Vis spectra of the AgNPs. UV-Vis spectra profile of AgNPs when exposed to different culture media, at $t=0 \mathrm{~h}$ and $\mathrm{t}=24 \mathrm{~h}$. 
Figure S2. UV-Vis profile from the centrifuged AgNPs supernatant
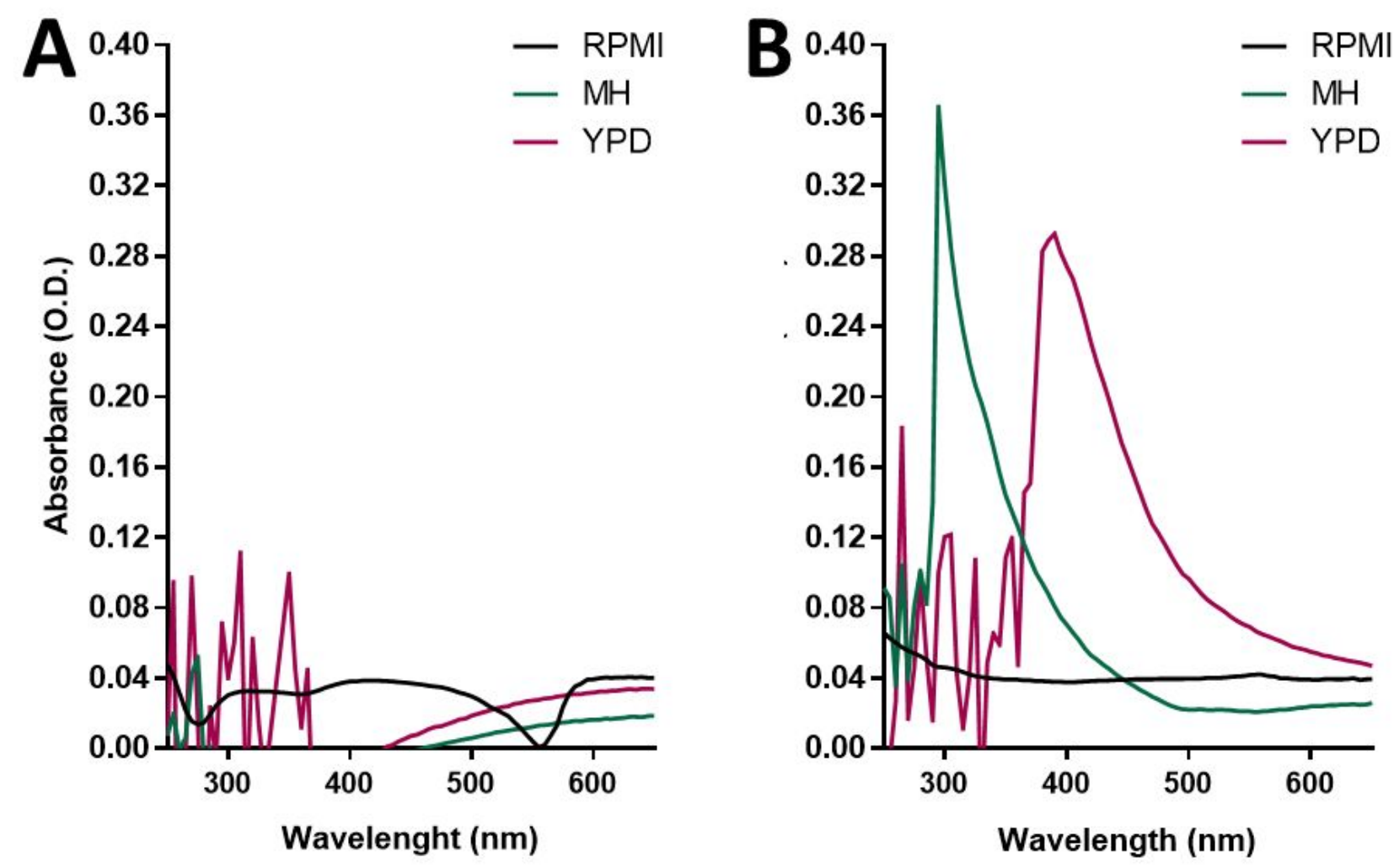

Figure S2. UV-Vis profile from the centrifuged AgNPs supernatant. The supernatant of the centrifuged AgNPs in RPMI, MH, and YPD showed different profiles at $t=0 \mathrm{~h}(\mathrm{~A})$ and $\mathrm{t}=24 \mathrm{~h}(\mathrm{~B})$. In all conditions, the absorbance profile reveals the potential presence of non-AgNPs silver species $\Lambda<300$ $\mathrm{nm}$. Only in YPD $\mathrm{t}=24 \mathrm{~h}$, a surface plasmon typical form the AgNPs was observed. 
Figure S3. Transmission electron micrographs of AgNPs used in this study.

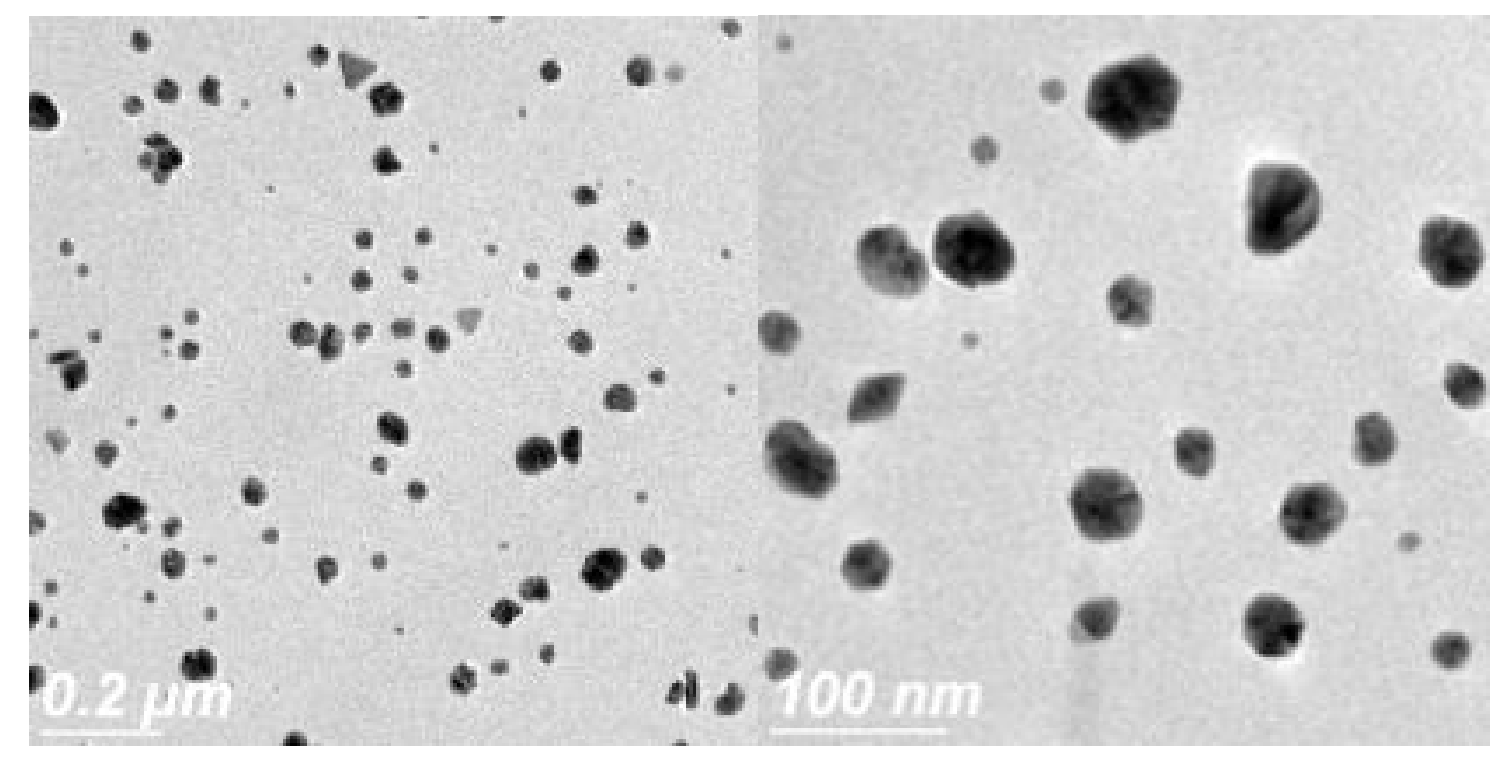

Figure S3. TEM micrographs of silver nanoparticles showed an aspect ratio close to 1 , with a metallic core diameter of $35 \pm 15 \mathrm{~nm}$. 
Figure S4. Size distribution of AgNPs in different culture media.
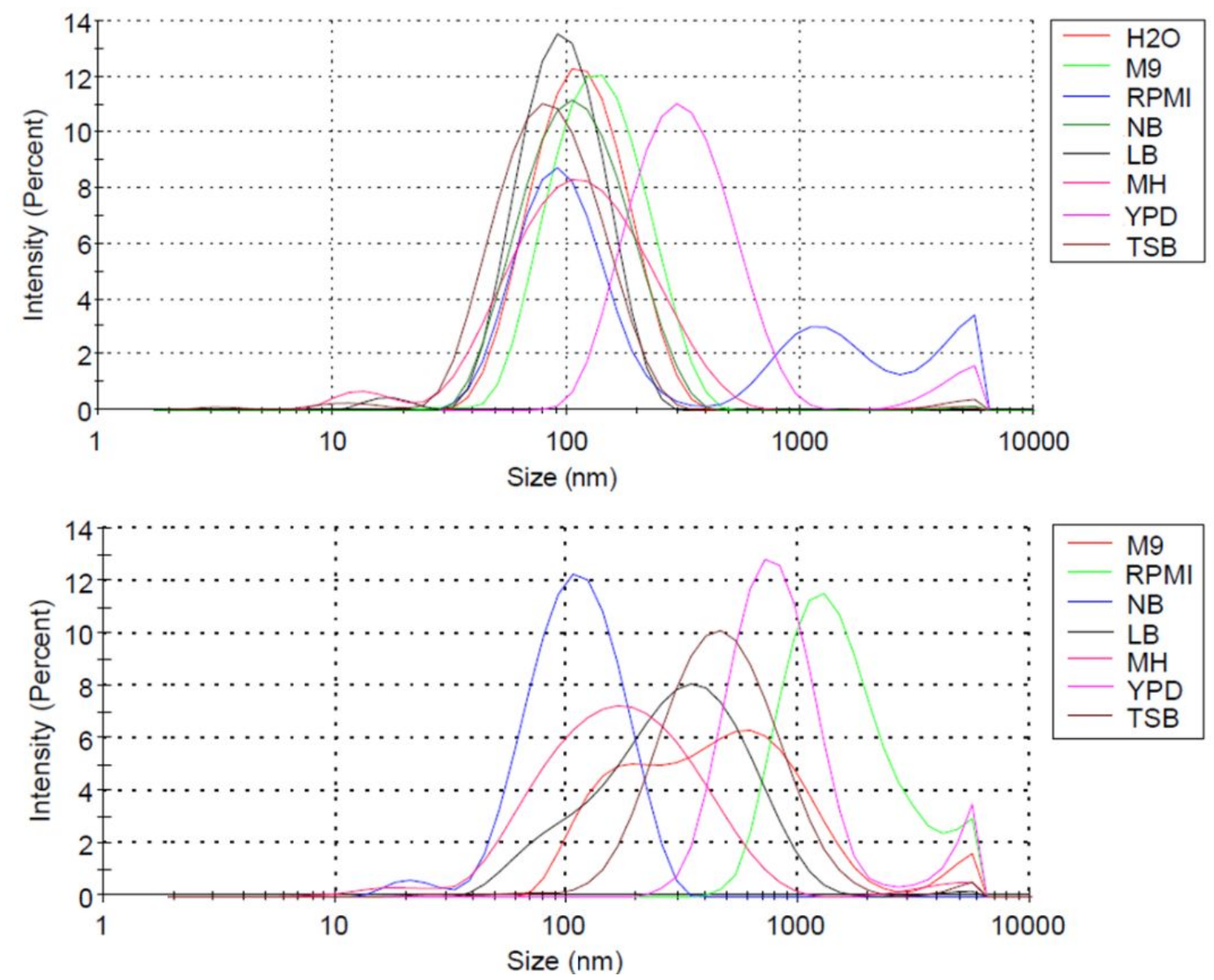

Figure S4. Hydrodynamic Size distribution graphs of AgNPs suspended in different culture media, at $\mathrm{t}=0 \mathrm{~h}$ (top) and $\mathrm{t}=24 \mathrm{~h}$ (bottom). The graphs reveal that AgNPs hydrodynamic size changes over time in different culture media. 
Table S1. DLS analysis.

Table S1. DLS characterization of AgNPs in different culture media

\begin{tabular}{|c|c|c|c|c|c|c|}
\hline \multirow{2}{*}{$\begin{array}{l}\text { Culture } \\
\text { media }\end{array}$} & \multicolumn{2}{|c|}{ Hydrodynamic size (nm) } & \multicolumn{2}{|c|}{ Polydispersity Index } & \multicolumn{2}{|c|}{ z-potential (mV) } \\
\hline & $t=0 h$ & $t=24 h$ & $t=0 h$ & $t=24 h$ & $\mathrm{t}=\mathbf{0 h}$ & $t=24 h$ \\
\hline Milli-Q $\mathrm{H}_{2} \mathrm{O}$ & 95.3 & 92.2 & 0.247 & 0.296 & -14.1 & -20.5 \\
\hline M9 & 124.5 & 332.4 & 0.196 & 0.443 & -16.5 & -17.9 \\
\hline RPMI & 136.2 & 784.2 & 0.621 & 0.327 & -16.9 & -16 \\
\hline NB & 87.8 & 215.1 & 0.266 & 0.222 & -14.3 & -10.8 \\
\hline LB & 77.7 & 90.1 & 0.229 & 0.404 & -10 & -16.1 \\
\hline $\mathrm{MH}$ & 89.8 & 113.6 & 0.369 & 0.417 & -8.16 & -24.4 \\
\hline YPD & 299.6 & 314 & 0.293 & 0.384 & -16.4 & -18.1 \\
\hline TSB & 67.4 & 373.5 & 0.291 & 0.315 & -14.8 & -15.6 \\
\hline
\end{tabular}


Table S2. Culture media broth major components.

Table S2. Culture media broth major components

\begin{tabular}{|c|c|c|c|c|c|}
\hline \multirow{2}{*}{$\begin{array}{c}\text { Culture } \\
\text { media }\end{array}$} & \multicolumn{4}{|c|}{ Grams per liter of solution (g/L) } & \multirow{2}{*}{$\mathrm{pH}$} \\
\hline & $\begin{array}{l}\text { Carbon } \\
\text { source }\end{array}$ & $\begin{array}{c}\text { Amino acids/ } \\
\text { proteins }\end{array}$ & $\begin{array}{c}\text { Inorganics } \\
\text { Salts }\end{array}$ & Others & \\
\hline M9 & 4 & 0 & 11.67 & $\begin{array}{l}\text { Biotin } .001 \\
\text { Trace elements } \\
\text { Thiamine } .001\end{array}$ & 7.5 \\
\hline RPMI * & 2 & 0.99 & 9.35 & $\begin{array}{l}\text { Vitamins } 0.04 \\
\text { Glutathione } 0.001 \\
\text { Phenol Red } 0.0053\end{array}$ & 7.2 \\
\hline NB & 0 \# & 8 & 0 \# & 0 \# & 6.8 \\
\hline LB & 0 \# & 15 & $5 \#$ & $0 \#$ & 7.5 \\
\hline $\mathrm{MH}$ & $1.5 \#$ & 19.5 & $0 \#$ & $0 \#$ & 7.4 \\
\hline YPD & 20 & 30 & 0 \# & $0^{\#}$ & 6.5 \\
\hline TSB & 2.5 \# & 20 & $7.5 \#$ & $0 \#$ & 7.3 \\
\hline
\end{tabular}




\section{Table S3. Silver content estimated by ICP-OES}

Table S3. The silver content varies over time in the different culture media

\begin{tabular}{ll|l}
\hline \multirow{2}{*}{ Culture media } & \multicolumn{2}{c}{ Silver content (ppm) } \\
& $\mathbf{t}=\mathbf{0} \mathbf{~}$ & $\mathbf{t}=\mathbf{2 4} \mathbf{~ h}$ \\
\hline RPMI & 0.34 & 0.17 \\
MH & 1.16 & 0.89 \\
YPD & 1.41 & 1.70
\end{tabular}




\section{Table S4. AgNPs MIC on E. coli, on different culture media}

Table S4. AgNPs MIC on E. coli, on different culture media, as determined by our study and the meta-analysis of the literature.

\begin{tabular}{|c|c|c|c|c|}
\hline \multirow{3}{*}{$\begin{array}{l}\text { Culture } \\
\text { media }\end{array}$} & \multicolumn{3}{|c|}{ MIC ranges $\left(\mu \mathrm{g} \mathrm{ml}^{-1}\right)^{\#}$} & \multirow{3}{*}{ References } \\
\hline & \multirow{2}{*}{ Our Data } & \multicolumn{2}{|c|}{ Data from literature } & \\
\hline & & All culture conditions ${ }^{\star *}$ & Similar conditions $\mathbf{s}^{* * *}$ & \\
\hline M9 & $N / A^{*}$ & $0.11-0.21$ & $0.11-0.21$ & 1,2 \\
\hline RPMI 1640 & 0.5 & 1.25 & 1.25 & 3 \\
\hline LB & 1 & $1-40$ & $1-40$ & $2,3,13-23$ \\
\hline NB & 2.5 & $0.25-180$ & $1-8$ & $4-12$ \\
\hline $\mathrm{MH}$ & 12.5 & $0.78-31.25$ & $1.6-31.25$ & $1,22,24-34$ \\
\hline TSB & 15 & $27-100$ & - & 22,35 \\
\hline $\mathrm{BHI}$ & $N / A^{*}$ & $0.5-2$ & $1.7-2$ & $36-38$ \\
\hline
\end{tabular}

\# We provide the MIC values published, still, most reports do not specify if those values are based on the total concentration of AgNPs or only to the silver content.

* The AgNPs MIC values for M9 and BHI were not determined.

** For "All culture conditions" we mean all the MIC values, regardless of initial inoculum size, exposition time, and temperature.

*** For "similar conditions", we considered those similar to the CLSI A09 protocol with a wider range in the inoculum size: only MIC values, an initial inoculum of $10^{5}$ to $10^{6}$ cells $\mathrm{ml}^{-1}, 24 \mathrm{~h}$ cultures, at $37^{\circ} \mathrm{C}$. 


\section{List S1. Culture media broth formulations.}

The followed standards recipes for the different culture media are described next. These were used for the preparation of culture media. The quantities of components are for a 1-liter 1X solution and were diluted in 1 distilled water; $\mathrm{pH}$ was adjusted using $\mathrm{NaOH}$ or $\mathrm{HCL}$, as needed. Culture media was sterilized by autoclaving or filtration, following the seller recommendations.

\section{CULTURE MEDIA REAGENTS}

\section{M9 media}

M9 salt solution

- $\quad \mathrm{Na} 2 \mathrm{HPO} 43.37 \mathrm{mM}(7.52 \mathrm{~g} / \mathrm{L})$

- $\mathrm{KH} 2 \mathrm{PO} 42.20 \mathrm{mM}(3 \mathrm{~g} / \mathrm{L})$

- $\quad \mathrm{NaCl} 0.855 \mathrm{mM}(0.5 \mathrm{~g} / \mathrm{L})$

- $\mathrm{NH} 4 \mathrm{Cl} 0.935 \mathrm{mM}(0.5 \mathrm{~g} / \mathrm{L})$

Glucose

$1 \mathrm{mM} \mathrm{MgSO} 4$

$0.3 \mathrm{mM} \mathrm{CaCl} 2$

Biotin

Thiamin

Trace elements

$\mathrm{pH} 7.5$

\section{QUANTITY}

$11.52 \mathrm{~g}$

\section{$4 \mathrm{~g}$}

$120 \mathrm{mg}$

$33 \mathrm{mg} / \mathrm{L}$

$1 \mu \mathrm{g}$

$1 \mu \mathrm{g}$

$0.1 \%$

\section{RPMI 1640 (R0883)}

Inorganic Salts

- Calcium Nitrate $\cdot 4 \mathrm{H} 2 \mathrm{O}(0.1 \mathrm{~g})$

- Magnesium Sulfate (anhydrous) (0.05 g)

- Potassium Chloride $(0.4 \mathrm{~g})$

- Sodium Bicarbonate $(2 \mathrm{~g})$

- Sodium Chloride (6 g)

- Sodium Phosphate Dibasic (anhydrous) (0.8 g)

Amino Acids

- L-Alanyl-L-Glutamine (0)

- L-Arginine (0.2)

- L-Asparagine (anhydrous) (0.05)

- L-Aspartic Acid (0.02)

- L-Cystine $\cdot 2 \mathrm{HCl}(0.0652)$

- L-Glutamic Acid (0.02)

- L-Glutamine (-)

- Glycine (0.01)

- L-Histidine (0.015)

- Hydroxy-L-Proline (0.02)

- L-Isoleucine (0.05)

- L-Leucine (0.05) 
- $\quad$ L-Lysine $\cdot \mathrm{HCl}(0.04)$

- L-Methionine (0.015)

- L-Phenylalanine (0.015)

- L-Proline (0.02)

- L-Serine

- L-Threonine (0.02)

- L-Tryptophan (0.005)

- $\quad$ L-Tyrosine $\cdot 2 \mathrm{Na} \cdot 2 \mathrm{H}_{2} \mathrm{O}(0.02883)$

- L-Valine (0.02)

Vitamins

- D-Biotin0.0002

- Choline Chloride 0.003

- Folic Acid 0.001

- myo-Inositol 0.035

- Niacinamide 0.001

- p-Aminobenzoic Acid 0.001

- D-Pantothenic Acid (hemicalcium) 0.00025

- Pyridoxine $\cdot \mathrm{HCl} \quad 0.001$

- Riboflavin 0.0002

- Thiamine $\cdot \mathrm{HCl} 0.001$

- Vitamin B12 0.000005

Other

- D-Glucose

2

- Glutathione (reduced)

0.001

- Phenol Red •Na

0.0053

- L-Glutamine

0.3

- Sodium Bicarbonate

0

$\mathrm{pH} 7.2 \pm 0.2$

\section{Nutrient Broth (NB)}

Beef Extract

Peptone

$\mathrm{pH} 6.8 \pm 0.2$

\section{Luria Bertani broth (LB)}

Peptone

Yeast Extract

Sodium Chloride

$\mathrm{pH} 7.5 \pm 0.2$

$5 \mathrm{~g}$

MH

Acid Digest of Casein

$\mathrm{pH} 7.4 \pm 0.2$ 
YPD

Yeast extract

$10 \mathrm{~g}$

Peptone

$20 \mathrm{~g}$

Dextrose (glucose)

$20 \mathrm{~g}$

$\mathrm{pH} 6.5 \pm 0.2$

\section{TSB}

Pancreatic Digest of Casein

$17 \mathrm{~g}$

Sodium Chloride

$5 \mathrm{~g}$

Papaic Digest of Soybean Meal

$3 \mathrm{~g}$

Dextrose

$2.5 \mathrm{~g}$

Dipotassium Phosphate

$2.5 \mathrm{~g}$

$\mathrm{pH} 7.3 \pm 0.2$ 


\section{References}

(1) Jadalannagari, S.; Deshmukh, K.; Ramanan, S. R.; Kowshik, M. Antimicrobial Activity of Hemocompatible Silver Doped Hydroxyapatite Nanoparticles Synthesized by Modified Sol-Gel Technique. Appl. Nanosci. 2014, 4 (2), 133-141. https://doi.org/10.1007/s13204-013-0197-x.

(2) Lok, C.-N.; Ho, C.-M.; Chen, R.; He, Q.-Y.; Yu, W.-Y.; Sun, H.; Tam, P. K.-H.; Chiu, J.-F.; Che, C.-M. Silver Nanoparticles: Partial Oxidation and Antibacterial Activities. JBIC J. Biol. Inorg. Chem. 2007, 12 (4), 527534. https://doi.org/10.1007/s00775-007-0208-z.

(3) Peetsch, A.; Greulich, C.; Braun, D.; Stroetges, C.; Rehage, H.; Siebers, B.; Köller, M.; Epple, M. SilverDoped Calcium Phosphate Nanoparticles: Synthesis, Characterization, and Toxic Effects toward Mammalian and Prokaryotic Cells. Colloids Surfaces B Biointerfaces 2013, 102, 724-729. https://doi.org/10.1016/J.COLSURFB.2012.09.040.

(4) Tahir, K.; Ahmad, A.; Li, B.; Nazir, S.; Khan, A. U.; Nasir, T.; Khan, Z. U. H.; Naz, R.; Raza, M. Visible Light Photo Catalytic Inactivation of Bacteria and Photo Degradation of Methylene Blue with $\mathrm{Ag} / \mathrm{TiO} 2$ Nanocomposite Prepared by a Novel Method. J. Photochem. Photobiol. B Biol. 2016, 162, 189-198. https://doi.org/10.1016/j.jphotobiol.2016.06.039.

(5) Radzig, M. A.; Nadtochenko, V. A.; Koksharova, O. A.; Kiwi, J.; Lipasova, V. A.; Khmel, I. A. Antibacterial Effects of Silver Nanoparticles on Gram-Negative Bacteria: Influence on the Growth and Biofilms Formation, Mechanisms of Action. Colloids Surfaces B Biointerfaces 2013, 102, 300-306. https://doi.org/10.1016/j.colsurfb.2012.07.039.

(6) Liu, H.-L.; Dai, S. A.; Fu, K.-Y.; Hsu, S.-H. Antibacterial Properties of Silver Nanoparticles in Three Different Sizes and Their Nanocomposites with a New Waterborne Polyurethane. Int. J. Nanomedicine 2010, 5, 10171028. https://doi.org/10.2147/IJN.S14572.

(7) Xia, Q.; Ma, Y.; Wang, J.; Xia, Q. H.; Ma, Y. J.; Wang, J. W. Biosynthesis of Silver Nanoparticles Using Taxus Yunnanensis Callus and Their Antibacterial Activity and Cytotoxicity in Human Cancer Cells. Nanomaterials 2016, 6 (9), 160. https://doi.org/10.3390/nano6090160.

(8) Ninganagouda, S.; Rathod, V.; Singh, D.; Hiremath, J.; Singh, A. K.; Mathew, J.; ul-Haq, M. Growth Kinetics and Mechanistic Action of Reactive Oxygen Species Released by Silver Nanoparticles from Aspergillus Niger on Escherichia Coli. Biomed Res. Int. 2014, 2014, 753419. https://doi.org/10.1155/2014/753419.

(9) Pal, S.; Tak, Y. K.; Song, J. M. Does the Antibacterial Activity of Silver Nanoparticles Depend on the Shape of the Nanoparticle? A Study of the Gram-Negative Bacterium Escherichia Coli. Appl. Environ. Microbiol. 2007, 73 (6), 1712-1720. https://doi.org/10.1128/AEM.02218-06.

(10) Agnihotri, S.; Mukherji, S.; Mukherji, S. Size-Controlled Silver Nanoparticles Synthesized over the Range 5$100 \mathrm{Nm}$ Using the Same Protocol and Their Antibacterial Efficacy. RSC Adv. 2014, 4 (8), 3974-3983. https://doi.org/10.1039/C3RA44507K.

(11) Ruparelia, J. P.; Chatterjee, A. K.; Duttagupta, S. P.; Mukherji, S. Strain Specificity in Antimicrobial Activity of Silver and Copper Nanoparticles. Acta Biomater. 2008, 4 (3), 707-716. https://doi.org/10.1016/j.actbio.2007.11.006.

(12) Raffi, M.; Hussain, F.; Bhatti, T. M.; Akhter, J. I.; Hameed, A.; Hasan, M. M. Antibacterial Characterization of Silver Nanoparticles against E. Coli ATCC-15224; 2008; Vol. 24.

(13) Shrivastava, S.; Bera, T.; Roy, A.; Singh, G.; Ramachandrarao, P.; Dash, D. Characterization of Enhanced Antibacterial Effects of Novel Silver Nanoparticles. Nanotechnology 2007, 18 (22), 225103. https://doi.org/10.1088/0957-4484/18/22/225103.

(14) Fayaz, A. M.; Balaji, K.; Girilal, M.; Yadav, R.; Kalaichelvan, P. T.; Venketesan, R. Biogenic Synthesis of Silver Nanoparticles and Their Synergistic Effect with Antibiotics: A Study against Gram-Positive and GramNegative Bacteria. Nanomedicine Nanotechnology, Biol. Med. 2010, 6 (1), 103-109. https://doi.org/10.1016/j.nano.2009.04.006.

(15) Li, P.; Li, J.; Wu, C.; Wu, Q.; Li, J. Synergistic Antibacterial Effects of $\beta$-Lactam Antibiotic Combined with Silver Nanoparticles. Nanotechnology 2005, 16 (9), 1912-1917. https://doi.org/10.1088/09574484/16/9/082.

(16) Zhang, Y.; Peng, H.; Huang, W.; Zhou, Y.; Yan, D. Facile Preparation and Characterization of Highly Antimicrobial Colloid Ag or Au Nanoparticles. J. Colloid Interface Sci. 2008, 325, 371-376. https://doi.org/10.1016/j.jcis.2008.05.063.

(17) Ivask, A.; Elbadawy, A.; Kaweeteerawat, C.; Boren, D.; Fischer, H.; Ji, Z.; Chang, C. H.; Liu, R.; Tolaymat, T.; Telesca, D.; Zink, J. I.; Cohen, Y.; Holden, P. A.; Godwin, H. A. Toxicity Mechanisms in Escherichia Coli Vary for Silver Nanoparticles and Differ from Ionic Silver. ACS Nano 2014, 8 (1), 374-386. 
Pallavicini, P.; Arciola, C. R.; Bertoglio, F.; Curtosi, S.; Dacarro, G.; D’Agostino, A.; Ferrari, F.; Merli, D.; Milanese, C.; Rossi, S.; Taglietti, A.; Tenci, M.; Visai, L. Silver Nanoparticles Synthesized and Coated with Pectin: An Ideal Compromise for Anti-Bacterial and Anti-Biofilm Action Combined with Wound-Healing Properties. J. Colloid Interface Sci. 2017, 498, 271-281. https://doi.org/10.1016/j.jcis.2017.03.062.

(19) Goswami, S. R.; Sahareen, T.; Singh, M.; Kumar, S. Role of Biogenic Silver Nanoparticles in Disruption of Cell-Cell Adhesion in Staphylococcus Aureus and Escherichia Coli Biofilm. J. Ind. Eng. Chem. 2015, 26, 73-80. https://doi.org/10.1016/J.JIEC.2014.11.017.

(20) Greulich, C.; Braun, D.; Peetsch, A.; Diendorf, J.; Siebers, B.; Epple, M.; Köller, M. The Toxic Effect of Silver Ions and Silver Nanoparticles towards Bacteria and Human Cells Occurs in the Same Concentration Range. RSC Adv. 2012, 2 (17), 6981. https://doi.org/10.1039/c2ra20684f.

(21) Li, D.; Liu, Z.; Yuan, Y.; Liu, Y.; Niu, F. Green Synthesis of Gallic Acid-Coated Silver Nanoparticles with High Antimicrobial Activity and Low Cytotoxicity to Normal Cells. Process Biochem. 2015, 50 (3), 357-366. https://doi.org/10.1016/J.PROCBIO.2015.01.002.

(22) Zhang, S.; Liu, L.; Pareek, V.; Becker, T.; Liang, J.; Liu, S. Effects of Broth Composition and Light Condition on Antimicrobial Susceptibility Testing of Ionic Silver. J. Microbiol. Methods 2014, 105, 42-46. https://doi.org/10.1016/J.MIMET.2014.07.009.

(23) Ansari, M. A.; Khan, H. M.; Khan, A. A.; Ahmad, M. K. Interaction of Silver Nanoparticles with Escherichia Coli and Their Cell Envelope Biomolecules. 2014, 905-915. https://doi.org/10.1002/jobm.201300457.

(24) Vazquez-Muñoz, R.; Borrego, B.; Juárez-Moreno, K.; García-García, M.; Mota Morales, J. D.; Bogdanchikova, N.; Huerta-Saquero, A. Toxicity of Silver Nanoparticles in Biological Systems: Does the Complexity of Biological Systems Matter? Toxicol. Lett. 2017, 276, 11-20. https://doi.org/10.1016/j.toxlet.2017.05.007.

(25) Vazquez-Muñoz, R.; Meza-Villezcas, A.; Fournier, P. G. J.; Soria-Castro, E.; Juarez-Moreno, K.; GallegoHernández, A. L.; Bogdanchikova, N.; Vazquez-Duhalt, R.; Huerta-Saquero, A. Enhancement of Antibiotics Antimicrobial Activity Due to the AgNPs Impact on the Cell Membrane. PLoS One 2019.

(26) Nogueira, S. S.; de Araujo-Nobre, A. R.; Mafud, A. C.; Guimarães, M. A.; Alves, M. M. M.; Plácido, A.; Carvalho, F. A. A.; Arcanjo, D. D. R.; Mascarenhas, Y.; Costa, F. G.; Albuquerque, P.; Eaton, P.; de Souza de Almeida Leite, J. R.; da Silva, D. A.; Cardoso, V. S. Silver Nanoparticle Stabilized by Hydrolyzed Collagen and Natural Polymers: Synthesis, Characterization and Antibacterial-Antifungal Evaluation. Int. J. Biol. Macromol. 2019, 135, 808-814. https://doi.org/10.1016/j.ijbiomac.2019.05.214.

(27) Orlowski, P.; Zmigrodzka, M.; Tomaszewska, E.; Ranoszek-Soliwoda, K.; Czupryn, M.; Antos-Bielska, M.; Szemraj, J.; Celichowski, G.; Grobelny, J.; Krzyzowska, M. Tannic Acid-Modified Silver Nanoparticles for Wound Healing: The Importance of Size. Int. J. Nanomedicine 2018, 13, 991-1007. https://doi.org/10.2147/IJN.S154797.

(28) Annadhasan, M.; SankarBabu, V. R.; Naresh, R.; Umamaheswari, K.; Rajendiran, N. A Sunlight-Induced Rapid Synthesis of Silver Nanoparticles Using Sodium Salt of N-Cholyl Amino Acids and Its Antimicrobial Applications. Colloids Surfaces B Biointerfaces 2012, 96, 14-21. https://doi.org/10.1016/J.COLSURFB.2012.03.009.

(29) Kvítek, L.; Panáček, A.; Soukupová, J.; Kolář, M.; Večeřová, R.; Prucek, R.; Holecová, M.; Zbořil, R. Effect of Surfactants and Polymers on Stability and Antibacterial Activity of Silver Nanoparticles (NPs). 2008. https://doi.org/10.1021/jp711616v.

(30) Panáček, A.; Kvítek, L.; Smékalová, M.; Večeřová, R.; Kolář, M.; Röderová, M.; Dyčka, F.; Šebela, M.; Prucek, R.; Tomanec, O.; Zbořil, R. Bacterial Resistance to Silver Nanoparticles and How to Overcome It. Nat. Nanotechnol. 2018, 13 (1), 65-71. https://doi.org/10.1038/s41565-017-0013-y.

(31) Suchomel, P.; Kvitek, L.; Panacek, A.; Prucek, R.; Hrbac, J.; Vecerova, R.; Zboril, R. Comparative Study of Antimicrobial Activity of $\mathrm{AgBr}$ and $\mathrm{Ag}$ Nanoparticles (NPs). PLoS One 2015, 10 (3), 1-16. https://doi.org/10.1371/journal.pone.0119202.

(32) Martinez-Castanon, G. A.; Niño-Martínez, N.; Martínez-Gutierrez, F.; Martínez-Mendoza, J. R.; Ruiz, F. Synthesis and Antibacterial Activity of Silver Nanoparticles with Different Sizes. J. Nanoparticle Res. 2008, 10 (8), 1343-1348. https://doi.org/10.1007/s11051-008-9428-6.

(33) Prucek, R.; Tuček, J.; Kilianová, M.; Panáček, A.; Kvítek, L.; Filip, J.; Kolář, M.; Tománková, K.; Zbořil, R. The Targeted Antibacterial and Antifungal Properties of Magnetic Nanocomposite of Iron Oxide and Silver Nanoparticles. Biomaterials 2011, 32 (21), 4704-4713. https://doi.org/10.1016/j.biomaterials.2011.03.039.

(34) Li, W. R.; Xie, X. B.; Shi, Q. S.; Zeng, H. Y.; Ou-Yang, Y. S.; Chen, Y. Ben. Antibacterial Activity and Mechanism of Silver Nanoparticles on Escherichia Coli. Appl. Microbiol. Biotechnol. 2010, 85 (4), 1115- 
1122. https://doi.org/10.1007/s00253-009-2159-5.

(35) Kanmani, P.; Lim, S. T. Synthesis and Characterization of Pullulan-Mediated Silver Nanoparticles and Its Antimicrobial Activities. Carbohydr. Polym. 2013, $97 \quad$ (2), 421-428. https://doi.org/10.1016/j.carbpol.2013.04.048.

(36) Paredes, D.; Ortiz, C.; Torres, R. Synthesis, Characterization, and Evaluation of Antibacterial Effect of Ag Nanoparticles against Escherichia Coli O157:H7 and Methicillin- Resistant Staphylococcus Aureus (MRSA). Int. J. Nanomedicine 2014, 9 (1), 1717-1729. https://doi.org/10.2147/IJN.S57156.

(37) Martinez-Gutierrez, F.; Olive, P. L.; Banuelos, A.; Orrantia, E.; Nino, N.; Sanchez, E. M.; Ruiz, F.; Bach, H.; Av-Gay, Y. Synthesis, Characterization, and Evaluation of Antimicrobial and Cytotoxic Effect of Silver and Titanium Nanoparticles. Nanomedicine Nanotechnology, Biol. Med. 2010, 6 (5), 681-688. https://doi.org/10.1016/j.nano.2010.02.001.

(38) Martínez-Gutierrez, F.; Thi, E. P.; Silverman, J. M.; de Oliveira, C. C.; Svensson, S. L.; Hoek, A. Vanden; Sánchez, E. M.; Reiner, N. E.; Gaynor, E. C.; Pryzdial, E. L. G.; Conway, E. M.; Orrantia, E.; Ruiz, F.; AvGay, Y.; Bach, H. Antibacterial Activity, Inflammatory Response, Coagulation and Cytotoxicity Effects of Silver Nanoparticles. Nanomedicine Nanotechnology, Biol. Med. 2012, 8 (3), 328-336. https://doi.org/10.1016/j.nano.2011.06.014. 